\title{
Identifying Supernova Progenitors and Constraining the Explosion Channels
}

\author{
Schuyler D. Van Dyk \\ Spitzer Science Center, Caltech, 220-6, Pasadena, CA USA \\ email: vandyk@ipac.caltech.edu
}

\begin{abstract}
Connecting the endpoints of massive star evolution with the various types of corecollapse supernovae ( $\mathrm{SNe}$ ) is ultimately the fundamental puzzle to be explored and solved. We can assemble clues indirectly, e.g., from information about the environments in which stars explode and establish constraints on the evolutionary phases of these stars. However, this is best accomplished through direct identification of the actual star that has exploded in pre-supernova imaging, preferably in more than one photometric band, where color and luminosity for the star can be precisely measured. We can then interpret the star's properties in light of expectations from the latest massive stellar evolutionary models, to attempt to assign an initial mass to the progenitor. So far, this has been done most successfully for SNe II-P, for which we now know that red supergiants in a relatively limited initial mass range are responsible. More recently, we have limited examples of the progenitors of SNe II-L, IIn, and IIb. The progenitors of SNe Ib and Ic, however, have been elusive so far; I will discuss the current status of our knowledge of this particular channel.
\end{abstract}

Keywords. (stars:) supernovae: general, (stars:) supernovae: individual (SN 1987A, SN 1993J, SN 1999A, SN 1999br, SN 1999ev, SN 2002ap, SN 2003ie, SN 2003jg, SN 2004am, SN 2004dj, SN 2004et, SN 2004gt, SN 2005cs, SN 2005gl, SN 2006bc, SN 2007gr, SN 2008bk, SN 2008cn, SN 2009hd, SN 2009jf, SN 2009kr, SN 2010jl, SN 2011dh, SN 2012aw), stars: evolution, (stars:) supergiants, stars: Wolf-Rayet, galaxies: stellar content, (ISM:) dust, extinction

\section{Direct Identification of SN Progenitors}

The most satisfying way of determining which stars explode as which supernovae (SNe) is to directly identify the massive, pre-SN stellar progenitors. We can do this from groundbased imaging data for only the nearest galaxies (distances $d \lesssim 7 \mathrm{Mpc}$ ), the most famous example of which was the identification of $\mathrm{Sk}-69^{\circ} 202$ as the B3 supergiant progenitor of SN 1987A in the Large Magellanic Cloud (Arnett et al. 1989 and references therein). This is the best characterization of a progenitor so far, since not only did we have multicolor photometry for the star (Isserstedt 1975), but we also had an observed spectral type as well (Rousseau et al. 1978).

Most of the time we have to identify the progenitor stars in archival, high spatialresolution, Hubble Space Telescope (HST) images. This search is typically limited to SNe with $d \lesssim 20 \mathrm{Mpc}$, depending on the SN type, the intrinsic luminosity of its progenitor, and the stellar crowding in the SN environment. We have to hope that these pre-SN images contain the SN site and that they might be in more than one band. We initially identify progenitor candidates in the images by comparing with early-time, ground-based SN images. However, inevitably, we need higher-resolution HST SN images or images obtained with adaptive optics (AO) on large-aperture, ground-based telescopes.

Once we have the star identified, we attempt to characterize its intrinsic properties via the photometry from the images and estimates of the host galaxy distance, metallicity $(Z)$ at the SN site, and total extinction to the SN. We then compare these properties to 
theoretical expectations and attempt to map SN types and their progenitors to model predictions. So, what do the latest and greatest theoretical stellar evolutionary tracks predict and explain? For instance, Ekström et al. (2012) have produced massive-star models (for $M_{\text {ini }} \geqslant 7 M_{\odot}$ ) at solar $Z$ that are either non-rotating or include rotation. The rotating models agree better with the revised Humphreys-Davidson limit on the initial masses of red supergiants (RSGs) at $\sim 25 M_{\odot}$ (Levesque et al. 2005; Crowther 2007), and therefore are probably more realistic than non-rotating models. Other recent models have also included departures from the standardized mass-loss formulations. Yoon \& Cantiello (2010) computed models at solar $Z$ with pulsationally-driven superwinds during the red supergiant (RSG) phase, which strip the star more prior to explosion, resulting in the star being more yellow at terminus. Georgy (2012) also obtained more yellow supergiants (YSGs), also at solar $Z$, with arbitrarily-increased mass-loss rates used in the Geneva group models for $12-20 M_{\odot}$ stars. A special case is the evolution of the most massive stars; that Galactic Wolf-Rayet stars (WRs) have $M_{\mathrm{WR}} \lesssim 20 M_{\odot}$ (Crowther 2007) may entail continuum-driven luminous blue variable (LBV) eruptions which dramatically shed the star's outer envelope (Humphreys \& Davidson 1994; Smith \& Owocki 2006). Of course, all of these models are for single-star evolution — binarity has not been taken into account!

\section{Progenitors of the Various Supernova Types}

\subsection{Type $I I-P S N e$}

What are the progenitors of the most common core-collapse SNe, the Type II-Plateau (II-P)? There are "normal" SNe II-P, such as SN 1999em (Hamuy et al. 2001; Leonard et al. 2002; Elmhamdi et al. 2003; although, it may have been somewhat underluminous, relative to other examples of normal SNe II-P) and low-luminosity SNe II-P, e.g, SN 1999br in NGC 4900 (Pastorello et al. 2004) and SN 2005cs in M51 (Pastorello et al. 2009). The best example of a low-luminosity SN II-P so far is SN 2008bk in NGC 7793 (Fig. 1), at only 3.4 Mpc distance from us. In Van Dyk et al. (2012) we made the "second best" progenitor detection ever, in the form of accurate measurements of the RSG progenitor's spectral energy distribution (SED) at $V R I J H K$ from archival, ground-based Gemini and VLT imaging. We were able to fit this SED with a RSG model stellar atmosphere with $T_{\text {eff }}=3600 \mathrm{~K}$ (Gustafsson et al. 2008; and, assuming the extinction to the SN and progenitor were low, $\left.A_{V}=0.065 \mathrm{mag}\right)$. When this is done, we can estimate $M_{\text {ini }}=8$ $8.5 M_{\odot}$ for the progenitor via comparison with stellar tracks at subsolar metallicity. (See also Mattila et al. 2008.) The low luminosity from these events may arise from a low ${ }^{56} \mathrm{Ni}$ mass, produced in a shell around the core, rather than the core itself. This mass also intersects with that of the super-AGB stars, and there may be some dependency on low(er) $Z$ in the SN environment, which needs to be further explored.

So, what is the mass for the RSG progenitors of "normal" SNe II-P? This is not yet known. (Actually, at the time of this writing, the mass for the normal SN II-P 2012aw in Messier 95 was being estimated.) Smartt et al. (2009) attempted to estimate the initial mass range for all SNe II-P, including low- and high-luminosity ones. The problem is, not all of the SNe II-P assumed to be normal are normal, and not all of the SNe considered were, in all likelihood, SNe II-P. Seven of the SNe II-P considered were of low luminosity. SN 1999 ev and, particularly, SN 2004et (Maguire et al. 2010) were likely high-luminosity SNe II-P. SN 2003ie was possibly a peculiar SN II-P, similar to SNe 1999A and 1987A (Harutyunyan et al. 2008), the latter of which definitely had a BSG as progenitor (not a RSG). SN 2006bc is a probable SN II-Linear (II-L; Gallagher et al. 2010). Additionally, 
the initial mass estimates for both SN 2004dj and 2004am are constraints (not detections), based on the assumed turn-off masses of compact clusters, of which the progenitor stars were presumably members. So, both the $M_{\text {ini }}$ for normal SNe II-P and the range of $M_{\text {ini }}$ for all SNe II-P is still not known or well constrained.

What may provide some constraint on $M_{\text {ini }}$ for normal SNe II-P are the identifications of the progenitors of high-luminosity SNe II-P. Elias-Rosa et al. (2009) identified a yellow supergiant (YSG) with $M_{\mathrm{ini}}=15 \pm 2 M_{\odot}$ for SN $2008 \mathrm{cn}$ in pre-SN $H S T$ images of the host galaxy. This identification is for the most distant SN II-P so far, at $\sim 33 \mathrm{Mpc}$, so some caution should be exercised; Elias-Rosa et al. (2009) also consider the possibility that the detected star is really a close binary system. In particular, Van Dyk \& Jarrett (in prep.) have revisited the progenitor of SN 2004et in NGC 6946 (at 5.7 Mpc; much closer than SN 2008cn), for which Li et al. (2005) identified a massive YSG progenitor. Crockett et al. (2011) confirmed that the progenitor was a YSG, but assigned a far lower $M_{\text {ini }}$ for the star. However, Van Dyk \& Jarrett have included deep near-infrared $J H K_{s}$ pre-SN imaging of the host galaxy and find that the star's SED can be fit by a RSG stellar atmosphere with $T_{\text {eff }} \simeq 3600 \mathrm{~K}$, which, at subsolar metallicity in the SN environment, implies that the star had $M_{\text {ini }} \simeq 14-15 M_{\odot}$.

\subsection{Type $I I-L S N e$}

What about the progenitors of SNe II-L? Elias-Rosa et al. (2010) identified, again, a YSG progenitor for SN $2009 \mathrm{kr}$ in NGC 1832, with $M_{\mathrm{ini}}=18-24 M_{\odot}$ (Fraser et al. 2010 also consider this star to be a YSG, but assign a much lower $\left.M_{\text {ini }} \sim 15 M_{\odot}\right)$. A criticism one could make is that we have assigned the initial mass range by comparing to normal massive-star evolutionary tracks. However, the $M_{\mathrm{ini}}=20 M_{\odot}$ track from Yoon \& Cantiello (2010) terminates at almost exactly the same bolometric luminosity and

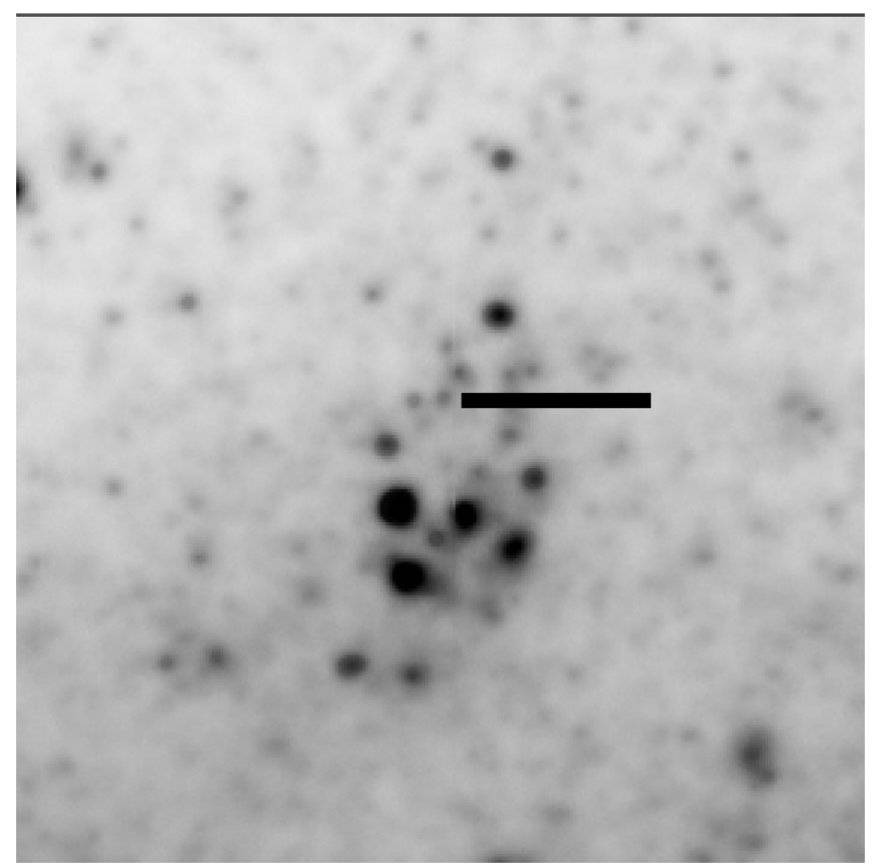

Figure 1. Gemini-S GMOS $g^{\prime} r^{\prime} i^{\prime}$ composite image from 2007, showing the location of the RSG progenitor star of the low-luminosity SN II-P 2008bk in NGC 7793, from Van Dyk et al. (2012). We consider this the "second best" SN progenitor detection ever (next to SN 1987A). 
effective temperature as the identified progenitor! So, it is quite possible that the yellow color results from enhanced pre-SN mass loss, which strips enough envelope to move the star from the RSG portion of the HR diagram. One other example of a SN II-L progenitor is the nearby SN 2009hd in Messier 66. Elias-Rosa et al. (2011) were able to detect the progenitor in pre-SN HST F814W images, but not in the corresponding, deep F555W images. This is almost certainly due to the high extinction $\left(A_{V} \approx 3.8 \mathrm{mag}\right)$. At best, Elias-Rosa et al. concluded the star was either a RSG or a YSG, with $M_{\text {ini }} \lesssim 20 M_{\odot}$.

\subsection{Type $I I b S N e$}

These are a hybrid of SNe II and SNe Ib, and the difference between SNe IIb and SNe Ib may be skin-deep. The best-known case to date is the SN IIb 1993J in Messier 81. The progenitor was an early K-type supergiant with $M_{\text {ini }} \sim 13-22 M_{\odot}$ (Aldering, Humphreys, \& Richmond 1994; Van Dyk et al. 2002). The progenitor's B supergiant companion was apparently detected in a very late-time, ground-based, optical spectrum (Maund et al. 2004). Another excellent, recent, nearby example is the SN IIb 2011dh in M51. The progenitor star was identified in deep HST/ACS multi-band images by both Van Dyk et al. (2011) and Maund et al. (2011). The latter authors claim that the F-type supergiant identified in the ACS images is the actual progenitor (see also Bersten, this volume). However, the early properties of the SN are most consistent with a compact progenitor (Arcavi et al. 2011; whereas SN 1993J clearly arose from an extended progenitor). So, Van Dyk et al. argue that the progenitor is an unseen, hot star (it was also not detected in HST/WFPC2 UV images) in an interacting binary system. When SN 2011dh has sufficiently faded, we can reimage the SN site with HST (or, far more likely, JWST!), to determine whether or not the yellow star is still there.

\subsection{Type IIn SNe}

The only relatively certain detection of a progenitor of this very heterogeneous SN type is the case of SN 2005gl in NGC 266 (at $d=66 \mathrm{Mpc}$ ). Gal-Yam et al. (2007) detected an object in a HST/WFPC2 F547M image from 1997, confirmed from the ground using Keck AO images of the SN. The astounding aspect is that the object had $M_{V} \approx-10.3$ mag (!!), which, among stars, only has a counterpart with something like a LBV in eruption. Gal-Yam \& Leonard (2009) later very likely confirmed that this object was the progenitor of SN 2005gl, in a HST/WFPC2 F547M image they obtained in 2007. The object had vanished! This is the first clear link of at least some SNe IIn to the explosion of LBVs. Other connections are more indirect, such as the modeling of SN 2006gy (Smith et al. 2007; Ofek et al. 2007) and indications from SNe IIn spectra (Kiewe et al. 2011).

Another example of a direct LBV-SN IIn connection may come from identification of the progenitor of SN 2010jl by Smith et al. (2011). They identified a luminous, blue object at the SN site, but it was unclear whether this was a single star or a compact star cluster. At the time of this writing, the SN continues to be too bright to determine whether or not the blue object is still at this position.

\subsection{Type $\mathrm{Ib} / \mathrm{c} \mathrm{SNe}$}

So far, no SN Ib or Ic progenitor has been directly identified. We (Van Dyk et al., in prep.) attempted to make this identification for SN Ib 2009jf in NGC 7479 (at $d=33.9$ Mpc, with extinction $A_{V}=0.53 \mathrm{mag}$ ), in HST/WFPC2 F569W and F814W images from 1995. We obtained similar HST/ACS F555W and F814W of the SN in 2010, when it had substantially faded. Unfortunately, no clear candidate for the progenitor was located; the SN was simply too distant from us. For this particular object, instead, we made a comparison of the bolometric light curve for SN 2009jf with the models by Dessart 
et al. (2011). The observed light curve is consistent with the model SN resulting from a close binary, with a primary star of $M_{\mathrm{ini}}=18 M_{\odot}$ which explodes as a nitrogen-rich WR $(\mathrm{WN})$ star with $M_{\text {final }}=3.79 M_{\odot}$; the secondary star in this model has $M_{\text {ini }}=17-23 M_{\odot}$ (Yoon, Woosley, \& Langer 2010).

For SNe Ic, one of the best examples may be SN 2004gt in NGC 4038, which occurred very near a star cluster in the host galaxy, such that both Gal-Yam et al. (2005) and Maund, Smartt, \& Schweizer (2005) only arrived at not very restrictive limits on the progenitor's nature, based on detection limits of the HST images and the inferred star cluster properties. Another similar example is the SN Ic $2007 \mathrm{gr}$ in NGC $1058(d=9.3$ $\mathrm{Mpc}, A_{V} \approx 0.3 \mathrm{mag}$ ), which also occurred very near, but still several half-light radii away from, a compact star cluster in the host. Of the other existing cases where progenitors could potentially be identified in HST images, many of these are in regions with very high extinction; hence, the progenitor was too extinguished to be identified. An example that we have worked on is the SN Ic 2003jg in NGC 2997, where the total extinction to the $\mathrm{SN}$ is estimated at $A_{V} \approx 4 \mathrm{mag}$.

Aggravating the problem further (in addition to the fact that nearby $\mathrm{SNe} \mathrm{Ib} / \mathrm{c}$ are comparatively rare) is that the progenitor is inferred theoretically to be quite blue (particularly, if it in at least some cases it is a single WR star), however, a dearth of (sufficiently deep) images obtained in blue or UV bands for nearby host galaxies exists in the HST archive. I illustrate this in Fig. 2, which shows what would be the apparent brightnesses of carbon-rich (WC) WRs at roughly the excitation temperature extremes, subtypes WC4 and WC8 (models from Sander, Hamann, \& Todt 2012), at typical distance moduli for host galaxies and possible total extinctions to the SN. The curves are the relevant HST instruments, WFPC2, WFC3, and ACS/WFC. What can be seen is that, in the redder bands, little hope exists in detecting WCs, unless the images happen to be very deep (limiting mags $\sim 26.5-29$ ), although the ground-based limit at $\sim V$ on the SN Ic 2002ap progenitor (Crockett et al. 2007) got very close. The best hope is for bands shortward of $\sim 4000 \AA$ A. Some potential exists for imaging, e.g., in the F336W band with WFC3, with total exposure times of $1800 \mathrm{~s}$ or deeper. However, one would have to match any detection of a progenitor with a detection in a corresponding band, either bluer or redder, to approximately the same depth, to derive color information for the star.

\section{Concluding Remarks}

We are dealing with small-number statistics here. It is absolutely essential that we continue to add to the numbers of SNe of all types with directly-identified progenitors, so that we can truly map SNe to the end states of stars of all possible initial masses.

Current evolutionary tracks do not adequately predict observed pre-SN stars. Once progenitor stars are directly identified in high spatial-resolution images, it is incumbent upon the theorists to produce models that explain the position of the star, in terms of its intrinsic properties, in the HR diagram. This is especially true for binary models for these progenitors.

I note that, like the monkeys in the famous wood carving in Nikko, I try to "hear no evil, see no evil, and speak no evil." But, hey, this is a competitive game!

Finally, I'd like to acknowledge the contributions of my several collaborators, but, particularly, Nancy Elias-Rosa (IEEC/CSIC, Spain), Alex Filippenko (UC Berkeley), and the late Weidong Li. 


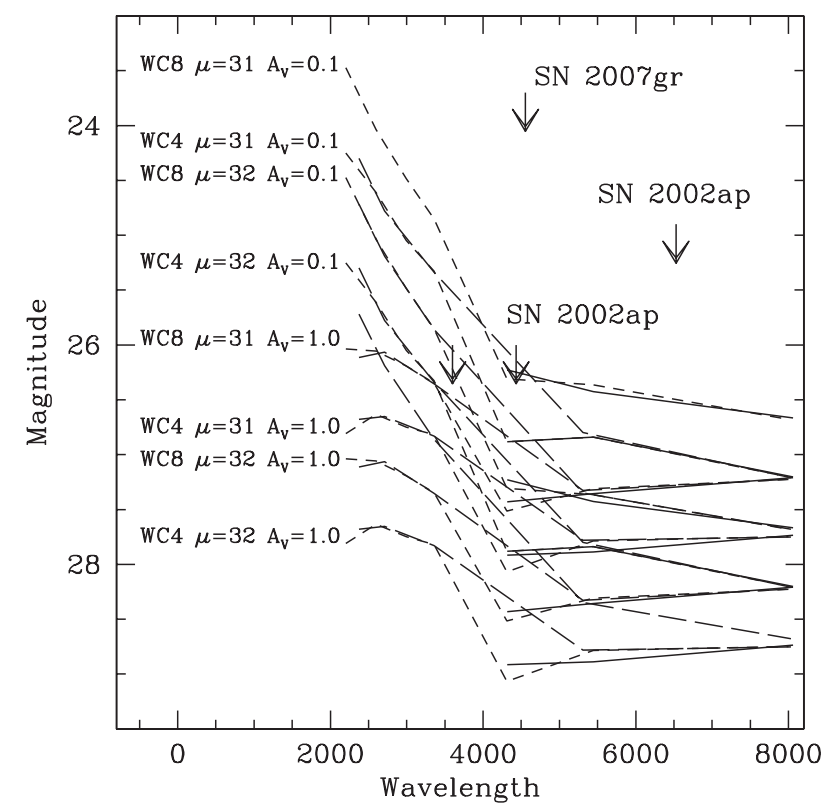

Figure 2. The SEDs of WC4 and WC8 stars from Sander et al. (2012) placed at typical distance moduli, $\mu=31$ and $32 \mathrm{mag}$, for host galaxies of SNe Ic, and further extinguished by $A_{V}=0.1$ or $1.0 \mathrm{mag}$. The various curves represent the available HST detectors, WFPC2 (short-dashed lines), WFC3 (long-dashed lines), and ACS/WFC (solid lines). Also shown are upper limits from published searches for SN Ic progenitors, Crockett et al. (2007) and Crockett et al. (2008), as well as the potential detection threshold for a 1800-s exposure with WFC3 through the F336W band.

\section{References}

Aldering, G., Humphreys, R. M., \& Richmond, M. 1994, AJ, 107, 662

Arcavi, I., Gal-Yam, A., Yaron, O., Sternberg, A., Rabinak, I., Waxman, E., Kasliwal, M. M., Quimby, R. M., Ofek, E. O., \& Horesh, A., et al. 2011, ApJ, 742, L18

Arnett, W. D., Bahcall, J. N., Kirshner, R. P., \& Woosley, S. E. 1989, ARAEAA, 27, 629

Crockett, R. M., Smartt, S. J., Eldridge, J. J., Mattila, S., Young, D. R., Pastorello, A., Maund, J. R., Benn, C. R., \& Skillen, I. 2007, MNRAS, 381, 835

Crockett, R. M., Maund, J. R., Smartt, S. J., Mattila, S., Pastorello, A., Smoker, J., Stephens, A. W., Fynbo, J., Eldridge, J. J., Danziger, I. J., \& Benn, C. R. 2008, ApJ, 672, L99

Crockett, R. M., Smartt, S. J., Pastorello, A., Eldridge, J. J., Stephens, A. W., Maund, J. R., \& Mattila, S. 2011, MNRAS, 410, 2767

Crowther, P. A. 2007, ARA\&A, 45, 177

Dessart, L., Hillier, D. J., Livne, E., Yoon, S.-C., Woosley, S., Waldman, R., \& Langer, N. 2011, MNRAS, 414, 2985

Ekström, S., Georgy, C., Eggenberger, P., Meynet, G., Mowlavi, N., Wyttenbach, A., Granada, A., Decressin, T., Hirschi, R., Frischknecht, U., Charbonnel, C., \& Maeder, A. 2012, A\&A, 537, A146

Elias-Rosa, N., Van Dyk, S. D., Li, W., Morrell, N., Gonzalez, S., Hamuy, M., Filippenko, A. V., Cuillandre, J.-C., Foley, R. J., \& Smith, N. 2009, ApJ, 706, 1174

Elias-Rosa, N., Van Dyk, S. D., Li, W., Miller, A. A., Silverman, J. M., Ganeshalingam, M., Boden, A. F., Kasliwal, M. M., Vinkó, J., Cuillandre, J.-C., et al. 2010, ApJ, 714, L254

Elias-Rosa, N., Van Dyk, S. D., Li, W., Silverman, J. M., Foley, R. J., Ganeshalingam, M., Mauerhan, J. C., Kankare, E., Jha, S., Filippenko, A. V., et al. 2011, ApJ, 742, 6

Fraser, M., Takts, K., Pastorello, A., Smartt, S. J., Mattila, S., Botticella, M.-T., Valenti, S., Ergon, M., Sollerman, J., Arcavi, I., et al. 2010, ApJ, 714, L280 
Elmhamdi, A., Danziger, I. J., Chugai, N., Pastorello, A., Turatto, M., Cappellaro, E., Altavilla, G., Benetti, S., Patat, F., \& Salvo, M. 2003, MNRAS, 338, 939

Gallagher, J. S., Clayton, G., Andrews, J., Sugerman, B., Clem, J., Barlow, M., Ercolano, B., Fabbri, J., Wesson, R., Otsuka, M., \& Meixner, M. 2011, BAAS, 43, 337.22

Gal-Yam, A., Fox, D. B., Kulkarni, S. R., Matthews, K., Leonard, D. C., Sand, D. J., Moon, D.-S., Cenko, S. B., \& Soderberg, A. M. (2005), ApJ, 630, L29

Gal-Yam, A., Leonard, D. C., Fox, D. B., Cenko, S. B., Soderberg, A. M., Moon, D.-S., Sand, D. J., Caltech Core Collapse Program, Li, W., Filippenko, A. V., Aldering, G., \& Copin, Y. 2007, ApJ, 656, 372

Gal-Yam, A. \& Leonard, D. C. 2009, Nature, 458, 865

Georgy, C. 2012, Aध A, 538, L8

Gustafsson, B., Edvardsson, B., Eriksson, K., Jørgensen, U. G., Nordlund, A. A., \& Plez, B. 2008, A\&BA, 486, 951

Hamuy, M., Pinto, P. A., Maza, J., Suntzeff, N. B., Phillips, M. M., Eastman, R. G., Smith, R. C., Corbally, C. J., Burstein, D., Li, Yong, et al. 2001, ApJ, 558, 615

Harutyunyan, A. H., Pfahler, P., Pastorello, A., Taubenberger, S., Turatto, M., Cappellaro, E., Benetti, S., Elias-Rosa, N., Navasardyan, H., Valenti, S., et al. 2008, A\&BA, 488, 383

Humphreys, R. M. \& Davidson, K. 1994, PASP, 106, 1025

Isserstedt, J. 1975, A\&SAS, 19, 259

Kiewe, M., Gal-Yam, A., Arcavi, I., Leonard, D. C., Emilio Enriquez, J., Cenko, S. B., Fox, D. B., Moon, D.-S., Sand, D. J., \& Soderberg, A. M., The CCCP 2012, ApJ, 744, 10

Leonard, D. C., Filippenko, A. V., Gates, E. L., Li, W., Eastman, R. G., Barth, A. J., Bus, S. J., Chornock, R., Coil, A. L., Frink, S., et al. 2002, PASP, 114, 35

Levesque, E. M., Massey, P., Olsen, K. A. G., Plez, B., Josselin, E., Maeder, A., \& Meynet, G. 2005, ApJ, 628, 973

Li, W., Van Dyk, S. D., Filippenko, A. V., \& Cuillandre, J.-C. 2005, PASP, 117, 121

Maguire, K., Di Carlo, E., Smartt, S. J., et al. 2010, MNRAS, 404, 981

Mattila, S., Smartt, S. J., Eldridge, J. J., Maund, J. R., Crockett, R. M., \& Danziger, I. J. 2008, ApJ, 688, L91

Maund, J. R., Smartt, S. J., Kudritzki, R. P., Podsiadlowski, P., \& Gilmore, G. F. 2004, Nature, 427,129

Maund, J. R., Smartt, S. J., \& Schweizer, F. 2005, ApJ, 630, L29

Maund, J. R., Fraser, M., Ergon, M., Pastorello, A., Smartt, S. J., Sollerman, J., Benetti, S., Botticella, M.-T., Bufano, F., Danziger, I. J., et al. 2011, ApJ, 739, L37

Ofek, E. O., Cameron, P. B., Kasliwal, M. M., Gal-Yam, A., Rau, A., Kulkarni, S. R., Frail, D. A., Chandra, P., Cenko, S. B., Soderberg, A. M., \& Immler, S. 2007, ApJ, 659, L13

Pastorello, A., Zampieri, L., Turatto, M., et al. 2004, MNRAS, 347, 74

Pastorello, A., Valenti, S., Zampieri, L., et al. 2009, MNRAS, 394, 2266

Rousseau, J., Martin, N., Prévot, L., et al. 1978, A\&AS, 31, 243

Sander, A., Hamann, W.-R., \& Todt, H. 2012, A\&A, in press (arXiv:1201.6354)

Smartt, S. J., Eldridge, J. J., Crockett, R. M., \& Maund, J. R. 2009, MNRAS, 395, 1409

Smith, N. \& Owocki, S. P. 2006, ApJ, 645, L45

Smith, N., Li, W., Foley, R. J., Wheeler, J. C., Pooley, D., Chornock, R., Filippenko, A. V., Silverman, J. M., Quimby, R., Bloom, J. S., \& Hansen, C. 2007, ApJ, 666, 1116

Smith, N. Li, W., Miller, A. A., Silverman, J. M., Filippenko, A. V., Cuillandre, J.-C., Cooper, M. C., Matheson, T., \& Van Dyk, S. D. 2011, ApJ, 732, 63

Van Dyk, S. D., Garnavich, P. M., Filippenko, A. V., Höflich, P., Kirshner, R. P., Kurucz, R. L., \& Challis, P. 2002, PASP, 114, 1322

Van Dyk, S. D., Li, W., Cenko, S. B., Kasliwal, M. M., Horesh, A., Ofek, E. O., Kraus, A. L., Silverman, J. M., Arcavi, I., Filippenko, A. V., et al., 2011, ApJ, 741, L28

Van Dyk, S. D., Davidge, T., J., Elias-Rosa, N., Taubenberger, S., Li, W., Levesque, E. M., Howerton, S., Pignata, G., Morrell, N., Hamuy, M., \& Filippenko, A. V. 2012, AJ, 143, 19

Yoon, S.-C. \& Cantiello, M. 2010, ApJ, 717, L62

Yoon, S.-C., Woosley, S. E., \& Langer, N. 2010, ApJ, 725, 940 


\section{Discussion}

GAL-YAM: We as a community need to push for HST imaging of nearby galaxies in the blue-UV bands.

Nomoto: SNe II-L and II-b are both products of close binary evolution, in my opinion So, the binary evolutionary tracks in the HR diagram should be used for comparison with the observed progenitor candidates. Especially, the binary model for the progenitor of SN IIb 2011dh [M. Bersten's talk] nicely reached the location in the diagram of the observed yellow supergiant.

VAN DYK: I do agree that SNe IIb likely arise from close binaries, and we have observational evidence from the radio emission that at least one SN II-L, 1979C, may have arisen from an interacting, although not necessarily very close, binary system. Our contention with the SN 2011dh system is that the unseen, hot companion exploded, not the detected yellow supergiant.

Bersten: How is it possible to determine the $M_{\mathrm{ZAMS}}$ for a YSG star using single stellar evolutionary calculations? To obtain a YSG in single stellar calculations, it is necessary for large mass loss, but there is no clear mechanism for that. With hydrodynamic modeling it is possible to explain the early light curve of SN 2011dh, using an extended progenitor with the radius of a YSG $\left(R \sim 300 R_{\odot}\right)$.

VAN DYK: As to the first question, one has to use the tracks with enhanced mass loss, whether due to pulsational instability or some other driver; these are now beginning to get you close to the location of the observed stars in the HR diagram. I also agree that binaries could also potentially get one to the same spot, so such binary models should be calculated to produce YSG progenitors. As to the last point, we feel that the extended progenitor model for SN 2011dh is not consistent with the behavior of the early light curves. Only time will tell, when years from now we can reimage the SN site with HST and see if the YSG is still there (in whatever shape it's in) or not. 